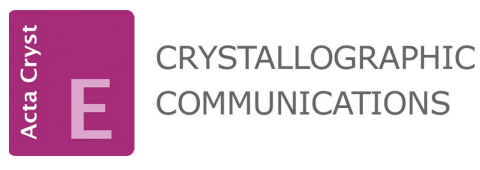

ISSN 2056-9890

Received 25 January 2021

Accepted 6 May 2021

Edited by D. Gray, University of Illinois UrbanaChampaign, USA

Keywords: crystal structure; hydrogen bond; $\mathrm{N}$ oxide; $\mathrm{C}-\mathrm{H} \cdots \pi$ (ring); nitrones; Hirshfeld surface analysis.

CCDC reference: 2082055

Supporting information: this article has supporting information at journals.iucr.org/e

\section{Crystal structure and Hirshfeld surface analysis of $\mathrm{N}$-[(Z)-(2-hydroxyphenyl)methylidene]aniline $N$-oxide}

Shaaban K. Mohamed, ${ }^{\mathrm{a}, \mathrm{b} *}$ Awad I. Said, ${ }^{\mathrm{c}}$ Joel T. Mague, ${ }^{\mathrm{d}}$ Moustafa F. Aly, ${ }^{\mathrm{e}}$ Mehmet Akkurt $^{\mathrm{f}}$ and Sahar M. I. Elgarhy ${ }^{\mathrm{g}}$

\begin{abstract}
${ }^{\mathbf{a} C}$ Chemistry and Environmental Division, Manchester Metropolitan University, Manchester, M1 5GD, England, ${ }^{\mathbf{b} C h e m i s t r y}$ Department, Faculty of Science, Minia University, 61519 El-Minia, Egypt, 'Chemistry Department, Faculty of Science, Assuit University, Egypt, ${ }^{\mathbf{d}}$ Department of Chemistry, Tulane University, New Orleans, LA 70118, USA, ${ }^{\mathbf{e}}$ Chemistry Department, Faculty of Science, South Valley University, Egypt, 'Department of Physics, Faculty of Sciences, Erciyes University, 38039 Kayseri, Turkey, and ${ }^{\mathrm{g}}$ Faculty of Science, Department of Bio Chemistry, Beni Suef University, Beni Suef, Egypt. *Correspondence e-mail: shaabankamel@yahoo.com
\end{abstract}

The conformation of the title compound, $\mathrm{C}_{13} \mathrm{H}_{11} \mathrm{NO}_{2}$, is partially determined by a strong, intramolecular $\mathrm{O}-\mathrm{H}$. . O hydrogen bond. The crystal packing consists of strongly corrugated layers parallel to the $a c$ plane and associated through $\mathrm{C}-$ $\mathrm{H} \cdots \pi$ (ring) interactions. A Hirshfeld surface analysis of the crystal structure indicates that the most significant contributions to the crystal packing are from $\mathrm{H} \cdots \mathrm{H}(44.1 \%), \mathrm{C} \cdots \mathrm{H} / \mathrm{H} \cdots \mathrm{C}(29.4 \%)$ and $\mathrm{O} \cdots \mathrm{H} / \mathrm{H} \cdots \mathrm{O}(17.3 \%)$ contacts.

\section{Chemical context}

Nitrones are a very important class of organic compounds as a result of their medicinal and pharmaceutical applications. They show antifungal (Salman et al., 2013), antibacterial (Chakraborty et al., 2010), neuroprotective (Chioua et al., 2012) and anticancer (Floyd et al., 2011) activities. In addition, nitrone compounds are widely used as antioxidant agents (AlMowali et al., 2014) because of their ability to scavenge free radicals. Based on these findings and following our interest in this area, we report herein the crystal structure of the title compound.

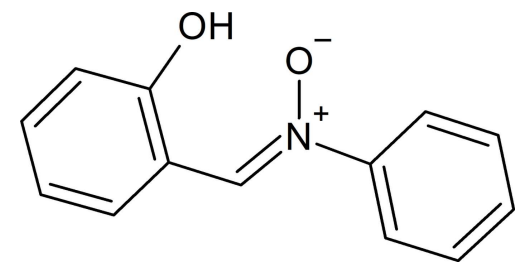

\section{Structural commentary}

The molecular structure of the title compound (Fig. 1) is almost planar, with maximum deviations of 0.398 (2) $\AA$ for O1 and -0.756 (2) $\AA$ for $\mathrm{O} 2$. The $\mathrm{N} 1-\mathrm{O} 2$ distance of 1.331 (2) $\AA$ is normal for a single bond and agrees well with those observed in other amine $N$-oxides. The dihedral angle between the aromatic rings (C1-C6 and C8-C13) is $1.94(12)^{\circ}$. The torsion angles $\mathrm{C} 2-\mathrm{C} 1-\mathrm{C} 7-\mathrm{N} 1, \mathrm{C} 1-\mathrm{C} 7-\mathrm{N} 1-\mathrm{C} 8$, $\mathrm{C} 1-\mathrm{C} 7-\mathrm{N} 1-\mathrm{O} 2, \mathrm{C} 7-\mathrm{N} 1-\mathrm{C} 8-\mathrm{C} 9$ and $\mathrm{O} 2-\mathrm{N} 1-\mathrm{C} 8-\mathrm{C}-9$ are $-30.2(3),-179.7(2),-0.4(3), 27.3(3)$ and $-152.0(2)^{\circ}$, respectively. The conformation of the title compound is partially determined by a strong, intramolecular O1$\mathrm{H} 1 \cdots \mathrm{O} 2$ hydrogen bond (Table 1). 


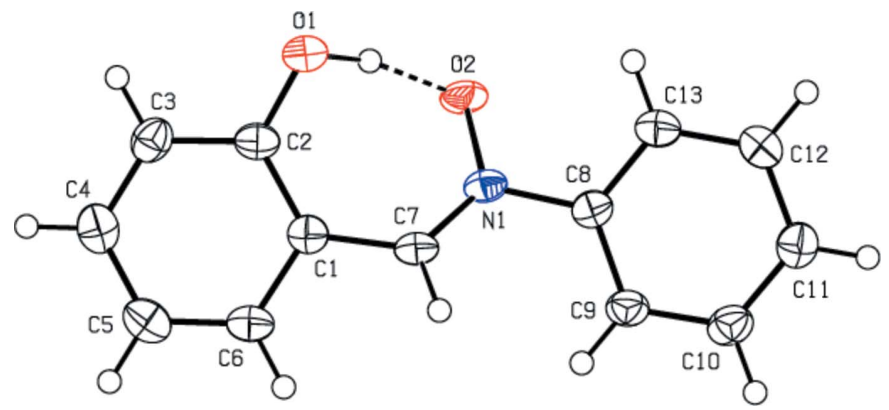

Figure 1

The title molecule with labelling scheme and $50 \%$ probability ellipsoids. The intramolecular hydrogen bond is shown by a dashed line.

\section{Supramolecular features}

In the crystal, $\mathrm{C} 7-\mathrm{H} 7 \cdots \mathrm{O} 2^{\mathrm{i}}$ hydrogen bonds (Table 1) link the molecules, forming chains along the $a$-axis direction. The chains are linked into strongly corrugated sheets parallel to the ac plane by $\mathrm{C} 10-\mathrm{H} 10 \cdots \mathrm{O} 2^{\mathrm{ii}}$ hydrogen bonds and $\mathrm{C} 11-$ $\mathrm{H} 11 \cdots C g 1^{1 i i}$ interactions $(\mathrm{Cg} 1$ is the centroid of the C1-C6 hydroxyphenyl ring; Table 1 and Fig. 2). The sheets are stacked along the $b$-axis direction by $\mathrm{C} 4-\mathrm{H} 4 \cdots \mathrm{Cg} 2^{\text {iv }}$ interactions ( $C g 2$ is the centroid of the $\mathrm{C} 8-\mathrm{C} 13$ phenyl ring; Table 1 and Figs. 2 and 3).

\section{Hirshfeld surface analysis}

A Hirshfeld surface analysis (Spackman \& Jayatilaka, 2009) was carried out using CrystalExplorer17.5 (Turner et al., 2017) to visualize the intermolecular interactions in the title compound. The Hirshfeld surface mapped over $d_{\text {norm }}$ (Fig. 4) shows the expected bright-red spots near atoms O1, O2, H7 and $\mathrm{H} 10$, which are involved in the $\mathrm{C}-\mathrm{H} \cdots \mathrm{O}$ hydrogenbonding interactions. The bright-red spot near $\mathrm{O} 1$ indicates its role as a hydrogen-bond acceptor to (C10)H10 (Fig. 4) and

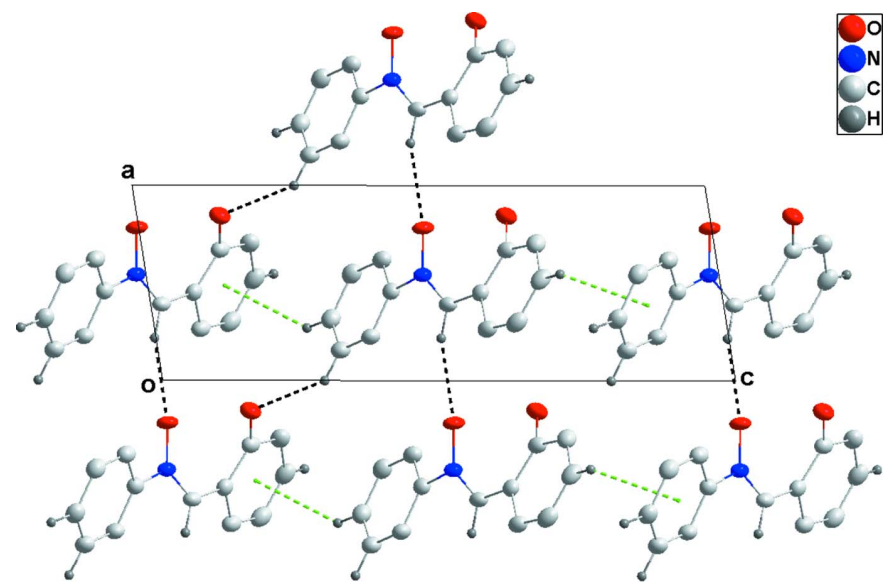

Figure 2

Detail of the intermolecular $\mathrm{C}-\mathrm{H} \cdots \mathrm{O}$ hydrogen bonds and the $\mathrm{C}-$ $\mathrm{H} \cdots \pi$ (ring) interactions (black and green dashed lines, respectively) viewed along the $b$-axis direction.
Table 1

Hydrogen-bond geometry $\left(\AA,^{\circ}\right)$.

$\mathrm{Cg} 1$ and $\mathrm{Cg} 2$ are the centroids of the $\mathrm{C} 1-\mathrm{C} 6$ and $\mathrm{C} 8-\mathrm{C} 13$ aromatic rings, respectively.

\begin{tabular}{lllll}
\hline$D-\mathrm{H} \cdots A$ & $D-\mathrm{H}$ & $\mathrm{H} \cdots A$ & $D \cdots A$ & $D-\mathrm{H} \cdots A$ \\
\hline $\mathrm{O} 1-\mathrm{H} 1 \cdots \mathrm{O} 2$ & 0.97 & 1.53 & $2.479(2)$ & 167 \\
$\mathrm{C} 7-\mathrm{H} 7 \cdots \mathrm{O} 2^{\mathrm{i}}$ & 0.95 & 2.43 & $3.368(3)$ & 167 \\
$\mathrm{C} 10-\mathrm{H} 10 \cdots \mathrm{O} 1^{\mathrm{ii}}$ & 0.95 & 2.53 & $3.227(3)$ & 131 \\
$\mathrm{C} 11-\mathrm{H} 11 \cdots C g 1^{\mathrm{iii}}$ & 0.95 & 2.94 & $3.662(3)$ & 136 \\
$\mathrm{C} 4-\mathrm{H} 4 \cdots C g 2^{\text {iv }}$ & 0.95 & 2.77 & $3.545(3)$ & 140 \\
\hline
\end{tabular}

Symmetry codes: (i) $x-1, y, z$; (ii) $\quad x-1,-y, z-\frac{1}{2}$; (iii) $x,-y, z-\frac{1}{2}$; (iv) $x,-y+1, z+\frac{1}{2}$.

Table 2

Summary of short interatomic contacts $(\AA)$ in the title compound.

\begin{tabular}{lll}
\hline Contact & Distance & Symmetry operation \\
\hline $\mathrm{O} 2 \cdots \mathrm{H} 7$ & 2.43 & $1+x, y, z$ \\
$\mathrm{O} 1 \cdots \mathrm{H} 10$ & 2.53 & $1+x,-y, \frac{1}{2}+z$ \\
$\mathrm{O} 2 \cdots \mathrm{H} 12$ & 2.87 & $x, 1+y, z$ \\
$\mathrm{C} 3 \cdots \mathrm{H} 12$ & 3.02 & $x,-y, \frac{1}{2}+z$ \\
$\mathrm{H} 4 \cdots \mathrm{C} 11$ & 2.86 & $x, 1-y, \frac{1}{2}+z$ \\
$\mathrm{H} 6 \cdots \mathrm{H} 13$ & 2.46 & $-1+x, 1+y, z$ \\
\hline
\end{tabular}

another red region near $\mathrm{O} 2$ correlates with the $\mathrm{C} 7-\mathrm{H} 7 \cdots \mathrm{O} 2$ interaction.

The two-dimensional fingerprint plots show the relative contributions of the various types of contacts to the Hirshfeld surface for the title compound (McKinnon et al., 2007). The plots (Fig. 5) reveal that $\mathrm{H} \cdots \mathrm{H}$ and $\mathrm{C} \cdots \mathrm{H} / \mathrm{H} \cdots \mathrm{C}$ interactions make the greatest contributions to the surface contacts, while $\mathrm{O} \cdots \mathrm{H} / \mathrm{H} \cdots \mathrm{O}, \mathrm{C} \cdots \mathrm{C}, \mathrm{N} \cdots \mathrm{H} / \mathrm{H} \cdots \mathrm{N}, \mathrm{N} \cdots \mathrm{C} / \mathrm{C} \cdots \mathrm{N}$ and $\mathrm{O} \cdots \mathrm{C} /$ C...O contacts are less significant (Tables 2 and 3).

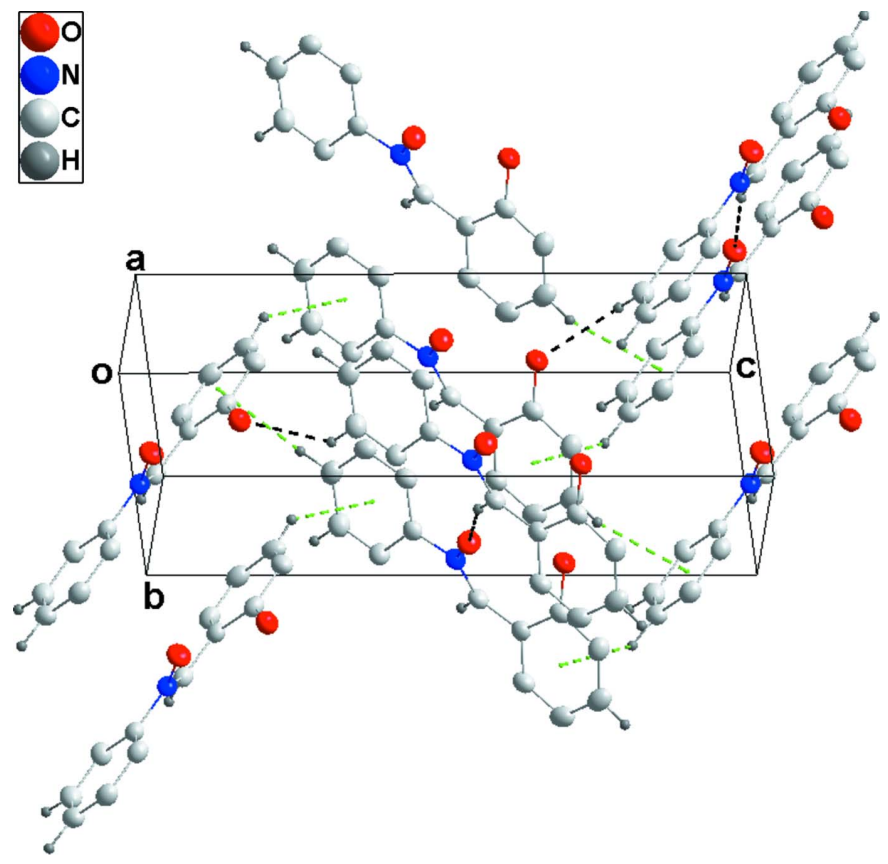

Figure 3

Packing viewed along the (120) direction with intermolecular interactions shown as in Fig. 2. 
Table 3

Percentage contributions of interatomic contacts to the Hirshfeld surface for the title compound.

\begin{tabular}{ll}
\hline Contact & Percentage contribution \\
\hline $\mathrm{H} \cdots \mathrm{H}$ & 44.1 \\
$\mathrm{C} \cdots \mathrm{H} / \mathrm{H} \cdots \mathrm{C}$ & 29.4 \\
$\mathrm{O} \cdots \mathrm{H} / \mathrm{H} \cdots \mathrm{O}$ & 17.3 \\
$\mathrm{C} \cdots \mathrm{C}$ & 5.3 \\
$\mathrm{~N} \cdots \mathrm{C} / \mathrm{C} \cdots \mathrm{N}$ & 1.7 \\
$\mathrm{~N} \cdots \mathrm{H} / \mathrm{H} \cdots \mathrm{N}$ & 1.5 \\
$\mathrm{O} \cdots \mathrm{C} / \mathrm{C} \cdots \mathrm{O}$ & 0.7 \\
\hline
\end{tabular}

\section{Database survey}

The four most closely related structures are $(Z)-N-[(1,3-$ diphenyl-1 $H$-pyrazol-4-yl)methanimine]- $N$-oxido (DEPVOM; Mohamed et al., 2018), (Z)-1,2-bis(3-bromophenyl)diazene 1-oxide (SIYHAK01; Goswami et al., 2018), (Z)- $N$-benzyl-

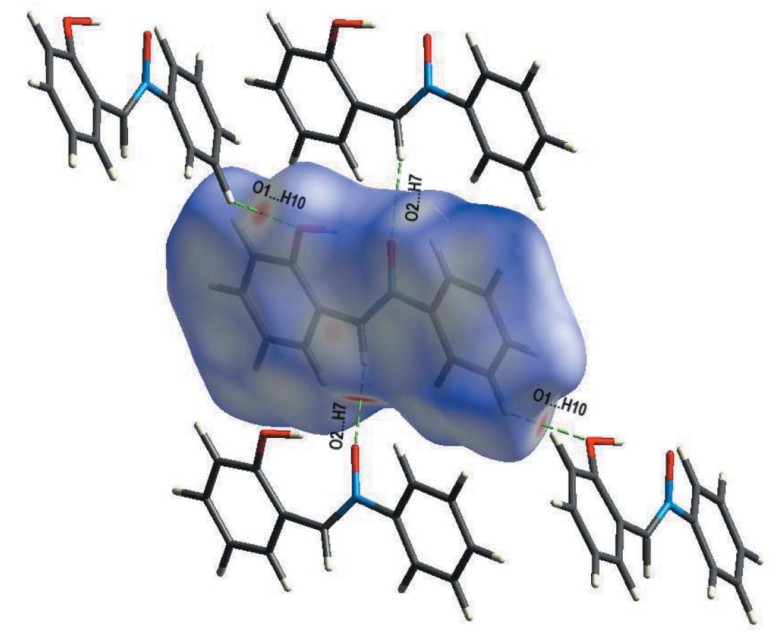

(a)

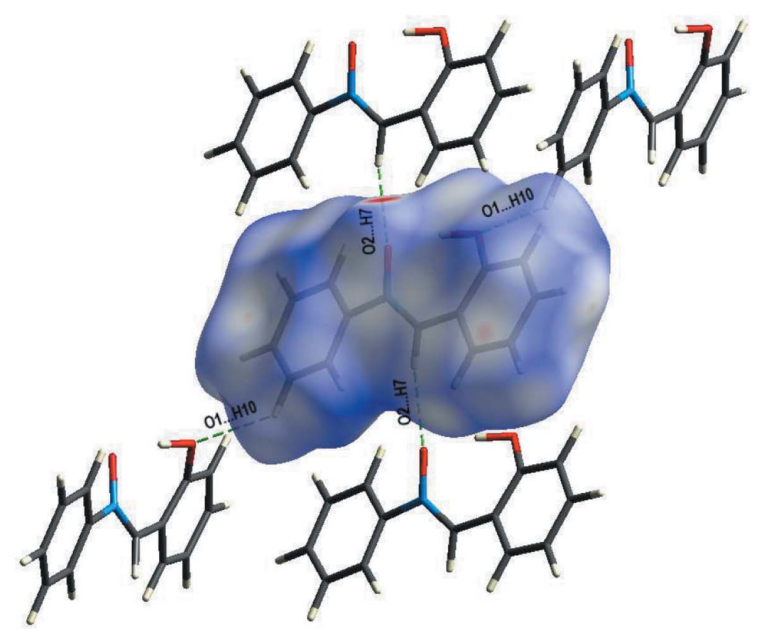

Figure 4

(b)

A view of the three-dimensional Hirshfeld surface with the $\mathrm{C}-\mathrm{H} \cdots \mathrm{O}$ interactions for the title compound, plotted over $d_{\text {norm }}$ in the range -0.2242 to 1.2146 a.u. (a) front view, $(b)$ back view.

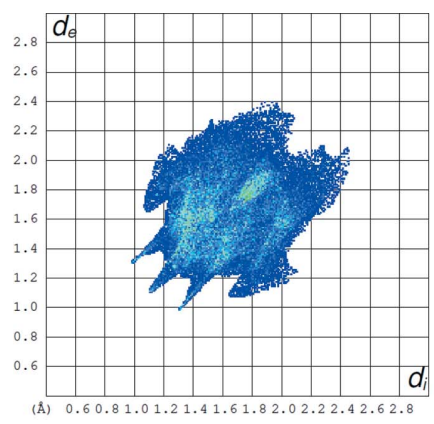

(a) All...All

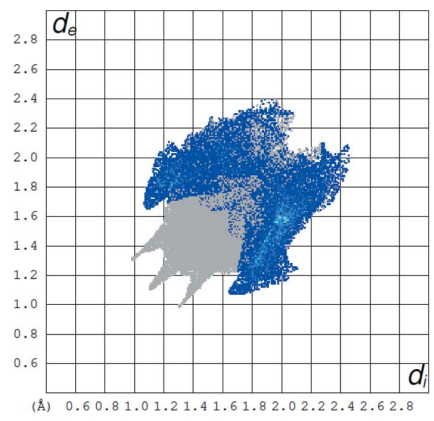

(c) $C \cdots H / H \cdots C$

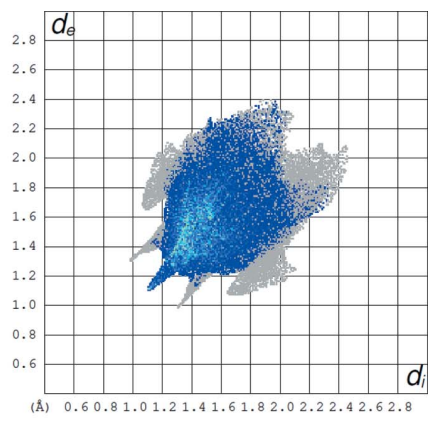

(b) $H \cdots H$

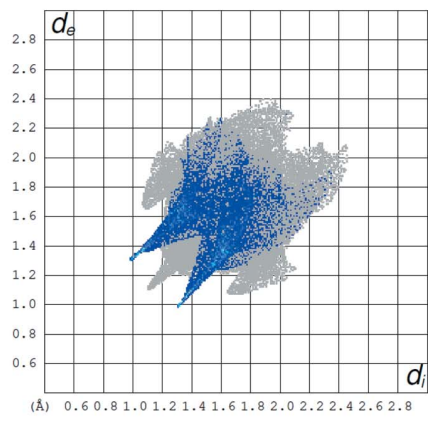

(d) $\mathrm{O} \cdots H / H \cdots O$
Figure 5

A view of the two-dimensional fingerprint plots for the title compound, showing (a) all interactions, and delineated into $(b) \mathrm{H} \cdots \mathrm{H},(c) \mathrm{C} \cdots \mathrm{H} /$ $\mathrm{H} \cdots \mathrm{C}$ and $(d) \mathrm{O} \cdots \mathrm{H} / \mathrm{H} \cdots \mathrm{O}$ interactions. The $d_{\mathrm{i}}$ and $d_{\mathrm{e}}$ values are the closest internal and external distances (in $\AA$ ) from given points on the Hirshfeld surface.

idene-1-phenylmethanamine oxide hydrogen peroxide solvate (JELQOJ; Churakov et al., 2017) and (Z)- $N$-(2-chlorobenzylidene)aniline $N$-oxide (ERIXEJ; Fu et al., 2011).

In the crystal of DEPVOM, (101) layers are generated by $\mathrm{C}-\mathrm{H} \cdots \mathrm{O}$ hydrogen bonds coupled with $\mathrm{C}-\mathrm{H} \cdots \pi($ ring $)$ and offset $\pi-\pi$ stacking interactions. In the crystal of SIYHAK01, $\mathrm{C}-\mathrm{H} \cdots \mathrm{O}$ and $\mathrm{C}-\mathrm{H} \cdots \mathrm{Br}$ hydrogen bonds together with offset $\pi-\pi$ interactions stack the molecules along the $a$-axis direction. In the crystal of JELQOJ, the organic and peroxide molecules are linked through both peroxide $\mathrm{O}-\mathrm{H}$ donor groups to oxide $\mathrm{O}$-atom acceptors, giving one-dimensional chains extending along the $b$-axis direction. Weak intermolecular $\mathrm{C}-\mathrm{H} \cdots \mathrm{O}$ hydrogen-bonding interactions are also present. In the crystal of ERIXEJ, the molecule is stabilized by an intramolecular $\mathrm{C}-\mathrm{H} \cdots \mathrm{O}$ hydrogen bond. The geometry about the $\mathrm{C}=\mathrm{N}$ bond is $Z[\mathrm{C}-\mathrm{C}-\mathrm{N}-\mathrm{O}$ torsion angle $=$ $\left.-4.2(3)^{\circ}\right]$ and the phenyl and benzene rings are transoriented around the $\mathrm{C}=\mathrm{N}$ bond. The phenyl and benzene rings make a dihedral angle of $56.99(2)^{\circ}$.

\section{Synthesis and crystallization}

(Z)-(2-Hydroxyphenyl)methylidene]benzenimine $\quad N$-oxide (nitrone) was prepared according to the reported procedures (Mobinikhaledi et al., 2005). $0.7 \mathrm{ml}$ (6 mmol) of salicyaldehyde were added to a warmed solution of $0.8 \mathrm{~g}(6 \mathrm{mmol})$ 
Table 4

Experimental details.

\begin{tabular}{|c|c|}
\hline \multicolumn{2}{|l|}{ Crystal data } \\
\hline Chemical formula & $\mathrm{C}_{13} \mathrm{H}_{11} \mathrm{NO}_{2}$ \\
\hline$M_{\mathrm{r}}$ & 213.23 \\
\hline Crystal system, space group & Monoclinic, $P c$ \\
\hline Temperature $(\mathrm{K})$ & 150 \\
\hline$a, b, c(\AA)$ & $5.5391(1), 5.7873(2), 16.0859$ (4) \\
\hline$\beta\left(^{\circ}\right)$ & $99.067(1)$ \\
\hline$V\left(\AA^{6}\right)$ & $509.21(2)$ \\
\hline$Z$ & 2 \\
\hline Radiation type & $\mathrm{Cu} K \alpha$ \\
\hline$\mu\left(\mathrm{mm}^{-1}\right)$ & 0.77 \\
\hline Crystal size $(\mathrm{mm})$ & $0.19 \times 0.17 \times 0.15$ \\
\hline \multicolumn{2}{|l|}{ Data collection } \\
\hline Diffractometer & $\begin{array}{l}\text { Bruker D8 VENTURE PHOTON } \\
100 \text { CMOS }\end{array}$ \\
\hline Absorption correction & $\begin{array}{l}\text { Multi-scan (SADABS; Krause et } \\
\quad \text { al., 2015) }\end{array}$ \\
\hline$T_{\min }, T_{\max }$ & $0.77,0.89$ \\
\hline $\begin{array}{l}\text { No. of measured, independent and } \\
\text { observed }[I>2 \sigma(I)] \text { reflections }\end{array}$ & $3578,1654,1607$ \\
\hline$R_{\text {int }}$ & 0.023 \\
\hline$(\sin \theta / \lambda)_{\max }\left(\AA^{-1}\right)$ & 0.618 \\
\hline \multicolumn{2}{|l|}{ Refinement } \\
\hline$R\left[F^{2}>2 \sigma\left(F^{2}\right)\right], w R\left(F^{2}\right), S$ & $0.033,0.084,1.06$ \\
\hline No. of reflections & 1654 \\
\hline No. of parameters & 145 \\
\hline No. of restraints & 2 \\
\hline $\mathrm{H}$-atom treatment & $\mathrm{H}$-atom parameters constrained \\
\hline$\Delta \rho_{\max }, \Delta \rho_{\min }\left(\mathrm{e} \AA^{-3}\right)$ & $0.18,-0.18$ \\
\hline Absolute structure & $\begin{array}{l}\text { Flack } x \text { determined using } 611 \\
\quad \text { quotients }\left[\left(I^{+}\right)-\left(I^{-}\right)\right] /\left[\left(I^{+}\right)+\left(I^{-}\right)\right] \\
\quad \text { (Parsons et al., 2013). }\end{array}$ \\
\hline Absolute structure parameter & $0.30(13)$ \\
\hline
\end{tabular}

Computer programs: APEX3 and SAINT (Bruker, 2016), SHELXT (Sheldrick, 2015a), SHELXL (Sheldrick, 2015b), ORTEP-3 for Windows (Farrugia, 2012), DIAMOND (Brandenburg \& Putz, 2012) and PLATON (Spek, 2020).

$N$-phenylhydroxyamine in ethanol followed by stirring for 5 minutes, then standing at room temperature in the dark overnight gave the nitrone, which was recrystallized from ethanol in 53\% yield; m.p. 387-388 K.

\section{Refinement}

Crystal and refinement details are presented in Table 4. The $\mathrm{H}$ atom of the $\mathrm{OH}$ group was found in difference-Fourier maps, and its positional parameters were fixed using the AFIX 3 instruction in SHELXL and were refined with the isotropic displacement parameter $U_{\text {iso }}(\mathrm{H})=1.5 U_{\text {eq }}(\mathrm{O})$. The C-bound $\mathrm{H}$ atoms were positioned geometrically, with $\mathrm{C}-\mathrm{H}=0.95 \AA$, and constrained to ride on their parent atoms, with $U_{\text {iso }}(\mathrm{H})=$
$1.2 U_{\text {eq }}(\mathrm{C})$. Attempts to determine the absolute structure did not produce a definitive result, viz.: Flack $x=0.2$ (3) by classical fit to all intensities 0.30 (14) from 611 selected quotients (Parsons' method). A round of TWIN/BASF refinement gave $\mathrm{BASF}=0.2$ (4) with no improvement in the model.

\section{Funding information}

The support of NSF-MRI grant No. 1228232 for the purchase of the diffractometer and Tulane University for support of the Tulane Crystallography Laboratory are gratefully acknowledged.

\section{References}

Al-Mowali, A. H., Majeed, N. N. \& Abbas, A. F. (2014). Res. J. Pharm. Biol. Chem. Sci. 5, 2119-2123.

Brandenburg, K. \& Putz, H. (2012). DIAMOND, Crystal Impact GbR, Bonn, Germany.

Bruker (2016). APEX3 and SAINT. Bruker AXS, Inc., Madison, Wisconsin, USA.

Chakraborty, B., Schhetric, M., Kafly, S. \& Samanta, A. (2010). Indian J. Chem. Sect. B, 49, 209-215.

Chioua, M., Sucunza, D., Soriano, E., Hadjipavlou-Litina, D., Alcázar, A., Ayuso, I., Oset-Gasque, M. J., González, M. P., Monjas, L., Rodríguez-Franco, M. I., Marco-Contelles, J. \& Samadi, A. (2012). J. Med. Chem. 55, 153-168.

Churakov, A. V., Prikhodchenko, P. V., Medvedev, A. G. \& Mikhaylov, A. A. (2017). Acta Cryst. E73, 1666-1669.

Farrugia, L. J. (2012). J. Appl. Cryst. 45, 849-854.

Floyd, R. A, Chandru, H. K., He, T. \& Towner, R. (2011). Anticancer Agents Med. Chem. 11, 373-379.

Fu, Y., Liu, Y., Yang, Y. \& Chen, Y. (2011). Acta Cryst. E67, o1320.

Goswami, S. K., Hanton, L. R., McAdam, C. J., Moratti, S. C. \& Simpson, J. (2018). IUCrData, 3, x181486.

Krause, L., Herbst-Irmer, R., Sheldrick, G. M. \& Stalke, D. (2015). J. Appl. Cryst. 48, 3-10.

McKinnon, J. J., Jayatilaka, D. \& Spackman, M. A. (2007). Chem. Commun. pp. 3814-3816.

Mobinikhaledi, A., Foroughifar, N. \& Kalate, Z. (2005). Turk. J. Chem. 29, 147-152.

Mohamed, S. K., Mague, J. T., Akkurt, M., Said, A. I., Hawaiz, F. E. \& Elgarhy, S. M. I. (2018). IUCrData, 3, x180208.

Parsons, S., Flack, H. D. \& Wagner, T. (2013). Acta Cryst. B69, 249 259.

Salman, H. H. \& Majeed, N. N. (2013). J. Basrah Res. 39, 99-111.

Sheldrick, G. M. (2015a). Acta Cryst. A71, 3-8.

Sheldrick, G. M. (2015b). Acta Cryst. C71, 3-8.

Spackman, M. A. \& Jayatilaka, D. (2009). CrystEngComm, 11, 19-32.

Spek, A. L. (2020). Acta Cryst. E76, 1-11.

Turner, M. J., McKinnon, J. J., Wolff, S. K., Grimwood, D. J., Spackman, P. R., Jayatilaka, D. \& Spackman, M. A. (2017). CrystalExplorer17. The University of Western Australia. 


\section{supporting information}

Acta Cryst. (2021). E77, 596-599 [https://doi.org/10.1107/S2056989021004813]

\section{Crystal structure and Hirshfeld surface analysis of $\mathrm{N}$-[(Z)-(2-hydroxyphenyl)- methylidene]aniline $N$-oxide}

\section{Shaaban K. Mohamed, Awad I. Said, Joel T. Mague, Moustafa F. Aly, Mehmet Akkurt and}

Sahar M. I. Elgarhy

Computing details

Data collection: APEX3 (Bruker, 2016); cell refinement: SAINT (Bruker, 2016); data reduction: SAINT (Bruker, 2016); program(s) used to solve structure: SHELXT (Sheldrick, 2015a); program(s) used to refine structure: SHELXL (Sheldrick, 2015b); molecular graphics: ORTEP-3 for Windows (Farrugia, 2012) and DIAMOND (Brandenburg \& Putz, 2012); software used to prepare material for publication: PLATON (Spek, 2020).

$\mathrm{N}$-[(Z)-(2-Hydroxyphenyl)methylidene]aniline N-oxide

Crystal data

$\mathrm{C}_{13} \mathrm{H}_{11} \mathrm{NO}_{2}$

$M_{r}=213.23$

Monoclinic, $P c$

$a=5.5391(1) \AA$

$b=5.7873(2) \AA$

$c=16.0859$ (4) $\AA$

$\beta=99.067(1)^{\circ}$

$V=509.21(2) \AA^{3}$

$Z=2$

\section{Data collection}

Bruker D8 VENTURE PHOTON 100 CMOS diffractometer

Radiation source: INCOATEC I $\mu \mathrm{S}$ micro-focus source

Mirror monochromator

Detector resolution: 10.4167 pixels $\mathrm{mm}^{-1}$

$\omega$ scans

Absorption correction: multi-scan

(SADABS; Krause et al., 2015)

\section{Refinement}

Refinement on $F^{2}$

Least-squares matrix: full

$R\left[F^{2}>2 \sigma\left(F^{2}\right)\right]=0.033$

$w R\left(F^{2}\right)=0.084$

$S=1.06$

1654 reflections

145 parameters

2 restraints
$F(000)=224$

$D_{\mathrm{x}}=1.391 \mathrm{Mg} \mathrm{m}^{-3}$

$\mathrm{Cu} K \alpha$ radiation, $\lambda=1.54178 \AA$

Cell parameters from 3430 reflections

$\theta=5.6-72.4^{\circ}$

$\mu=0.77 \mathrm{~mm}^{-1}$

$T=150 \mathrm{~K}$

Block, yellow

$0.19 \times 0.17 \times 0.15 \mathrm{~mm}$

$T_{\min }=0.77, T_{\max }=0.89$

3578 measured reflections

1654 independent reflections

1607 reflections with $I>2 \sigma(I)$

$R_{\text {int }}=0.023$

$\theta_{\text {max }}=72.4^{\circ}, \theta_{\min }=5.6^{\circ}$

$h=-6 \rightarrow 6$

$k=-7 \rightarrow 7$

$l=-19 \rightarrow 19$

Hydrogen site location: mixed

$\mathrm{H}$-atom parameters constrained

$w=1 /\left[\sigma^{2}\left(F_{0}{ }^{2}\right)+(0.0441 P)^{2}+0.084 P\right]$

where $P=\left(F_{\mathrm{o}}^{2}+2 F_{\mathrm{c}}^{2}\right) / 3$

$(\Delta / \sigma)_{\max }<0.001$

$\Delta \rho_{\max }=0.18 \mathrm{e} \AA^{-3}$

$\Delta \rho_{\min }=-0.18$ e $\AA^{-3}$ 
Absolute structure: Flack $x$ determined using 611 quotients $\left[\left(I^{+}\right)-(I)\right] /\left[\left(I^{+}\right)+\left(I^{-}\right)\right]$(Parsons et al., 2013).

Absolute structure parameter: 0.30 (13)

\section{Special details}

Geometry. All esds (except the esd in the dihedral angle between two 1.s. planes) are estimated using the full covariance matrix. The cell esds are taken into account individually in the estimation of esds in distances, angles and torsion angles; correlations between esds in cell parameters are only used when they are defined by crystal symmetry. An approximate (isotropic) treatment of cell esds is used for estimating esds involving l.s. planes.

Fractional atomic coordinates and isotropic or equivalent isotropic displacement parameters $\left(\AA^{2}\right)$

\begin{tabular}{lllll}
\hline & $x$ & $y$ & $z$ & $U_{\text {iso }}^{*} / U_{\text {eq }}$ \\
\hline O1 & $0.8449(3)$ & $0.3532(3)$ & $0.64513(12)$ & $0.0342(4)$ \\
H1 & 0.841875 & 0.289527 & 0.589214 & $0.051^{*}$ \\
O2 & $0.7828(3)$ & $0.2215(3)$ & $0.49662(12)$ & $0.0338(4)$ \\
N1 & $0.5424(3)$ & $0.1864(3)$ & $0.48272(13)$ & $0.0267(4)$ \\
C1 & $0.4520(4)$ & $0.4953(4)$ & $0.57874(14)$ & $0.0260(5)$ \\
C2 & $0.6705(4)$ & $0.5169(4)$ & $0.63617(15)$ & $0.0280(5)$ \\
C3 & $0.7030(4)$ & $0.7093(4)$ & $0.68930(16)$ & $0.0328(5)$ \\
H3 & 0.852651 & 0.728354 & 0.726592 & $0.039^{*}$ \\
C4 & $0.5207(5)$ & $0.8717(4)$ & $0.68821(18)$ & $0.0348(5)$ \\
H4 & 0.545906 & 1.001606 & 0.724635 & $0.042^{*}$ \\
C5 & $0.3009(4)$ & $0.8470(4)$ & $0.63443(17)$ & $0.0349(6)$ \\
H5 & 0.174797 & 0.958261 & 0.634589 & $0.042^{*}$ \\
C6 & $0.2662(4)$ & $0.6611(4)$ & $0.58092(16)$ & $0.0304(5)$ \\
H6 & 0.114410 & 0.643651 & 0.544695 & $0.036^{*}$ \\
C7 & $0.3908(4)$ & $0.3082(4)$ & $0.51888(15)$ & $0.0270(5)$ \\
H7 & 0.222390 & 0.270734 & 0.504648 & $0.032^{*}$ \\
C8 & $0.4586(4)$ & $0.0034(4)$ & $0.42327(14)$ & $0.0263(5)$ \\
C9 & $0.2257(4)$ & $0.0070(4)$ & $0.37527(15)$ & $0.0304(5)$ \\
H9 & 0.116018 & 0.130115 & 0.380872 & $0.036^{*}$ \\
C10 & $0.1571(4)$ & $-0.1720(4)$ & $0.31930(17)$ & $0.0340(5)$ \\
H10 & -0.001169 & -0.171673 & 0.286384 & $0.041^{*}$ \\
C11 & $0.3176(5)$ & $-0.3521(4)$ & $0.31083(16)$ & $0.0327(5)$ \\
H11 & 0.269918 & -0.473587 & 0.271973 & $0.039^{*}$ \\
C12 & $0.5480(5)$ & $-0.3533(4)$ & $0.35956(18)$ & $0.0324(5)$ \\
H12 & 0.657396 & -0.477040 & 0.354374 & $0.039^{*}$ \\
C13 & $0.6199(4)$ & $-0.1748(4)$ & $0.41587(16)$ & $0.0301(5)$ \\
H13 & 0.778043 & -0.175337 & 0.448865 & $0.036^{*}$ \\
& & & &
\end{tabular}

Atomic displacement parameters $\left(\AA^{2}\right)$

\begin{tabular}{lllllll}
\hline & $U^{11}$ & $U^{22}$ & $U^{33}$ & $U^{12}$ & $U^{13}$ & $U^{23}$ \\
\hline O1 & $0.0260(9)$ & $0.0382(9)$ & $0.0363(9)$ & $0.0038(6)$ & $-0.0010(7)$ & $0.0001(7)$ \\
O2 & $0.0137(7)$ & $0.0461(9)$ & $0.0418(9)$ & $-0.0015(7)$ & $0.0047(7)$ & $-0.0028(8)$ \\
N1 & $0.0170(9)$ & $0.0340(9)$ & $0.0290(10)$ & $-0.0013(7)$ & $0.0031(7)$ & $0.0025(8)$ \\
C1 & $0.0253(12)$ & $0.0287(11)$ & $0.0250(12)$ & $-0.0010(8)$ & $0.0073(10)$ & $0.0032(8)$
\end{tabular}


supporting information

\begin{tabular}{lllllll} 
C2 & $0.0232(12)$ & $0.0314(11)$ & $0.0301(12)$ & $0.0004(8)$ & $0.0057(10)$ & $0.0066(9)$ \\
C3 & $0.0308(13)$ & $0.0359(12)$ & $0.0320(12)$ & $-0.0082(10)$ & $0.0059(10)$ & $-0.0024(10)$ \\
C4 & $0.0380(14)$ & $0.0300(11)$ & $0.0397(13)$ & $-0.0066(9)$ & $0.0166(11)$ & $-0.0022(10)$ \\
C5 & $0.0346(14)$ & $0.0328(12)$ & $0.0403(15)$ & $0.0032(9)$ & $0.0150(11)$ & $0.0065(10)$ \\
C6 & $0.0232(11)$ & $0.0362(12)$ & $0.0321(12)$ & $0.0026(9)$ & $0.0054(9)$ & $0.0071(10)$ \\
C7 & $0.0185(10)$ & $0.0327(11)$ & $0.0293(11)$ & $0.0009(8)$ & $0.0028(9)$ & $0.0049(9)$ \\
C8 & $0.0241(11)$ & $0.0292(11)$ & $0.0258(13)$ & $-0.0022(8)$ & $0.0044(10)$ & $0.0029(8)$ \\
C9 & $0.0218(11)$ & $0.0379(12)$ & $0.0316(13)$ & $0.0017(9)$ & $0.0053(10)$ & $-0.0012(9)$ \\
C10 & $0.0246(11)$ & $0.0448(13)$ & $0.0329(12)$ & $-0.0037(9)$ & $0.0052(10)$ & $-0.0013(11)$ \\
C11 & $0.0328(12)$ & $0.0340(12)$ & $0.0328(13)$ & $-0.0066(9)$ & $0.0096(10)$ & $-0.0021(10)$ \\
C12 & $0.0336(12)$ & $0.0307(11)$ & $0.0341(12)$ & $0.0044(9)$ & $0.0096(10)$ & $0.0023(10)$ \\
C13 & $0.0249(11)$ & $0.0350(12)$ & $0.0307(13)$ & $0.0025(8)$ & $0.0056(9)$ & $0.0053(9)$ \\
& & & & & & \\
\hline
\end{tabular}

Geometric parameters $\left(\AA,{ }^{\circ}\right)$

\begin{tabular}{|c|c|c|c|}
\hline $\mathrm{O} 1-\mathrm{C} 2$ & $1.345(3)$ & $\mathrm{C} 5-\mathrm{H} 5$ & 0.9500 \\
\hline $\mathrm{O} 1-\mathrm{H} 1$ & 0.9697 & C6- $\mathrm{H} 6$ & 0.9500 \\
\hline $\mathrm{O} 2-\mathrm{N} 1$ & $1.331(2)$ & $\mathrm{C} 7-\mathrm{H} 7$ & 0.9500 \\
\hline $\mathrm{N} 1-\mathrm{C} 7$ & $1.302(3)$ & $\mathrm{C} 8-\mathrm{C} 13$ & $1.382(3)$ \\
\hline $\mathrm{N} 1-\mathrm{C} 8$ & $1.454(3)$ & $\mathrm{C} 8-\mathrm{C} 9$ & 1.395 \\
\hline $\mathrm{C} 1-\mathrm{C} 2$ & $1.407(3)$ & $\mathrm{C} 9-\mathrm{C} 10$ & $1.386(4)$ \\
\hline $\mathrm{C} 1-\mathrm{C} 6$ & $1.411(3)$ & $\mathrm{C} 9-\mathrm{H} 9$ & 0.9500 \\
\hline $\mathrm{C} 1-\mathrm{C} 7$ & $1.453(3)$ & $\mathrm{C} 10-\mathrm{C} 11$ & $1.391(4)$ \\
\hline $\mathrm{C} 2-\mathrm{C} 3$ & $1.398(4)$ & $\mathrm{C} 10-\mathrm{H} 10$ & 0.9500 \\
\hline $\mathrm{C} 3-\mathrm{C} 4$ & $1.377(4)$ & $\mathrm{C} 11-\mathrm{C} 12$ & $1.389(4)$ \\
\hline $\mathrm{C} 3-\mathrm{H} 3$ & 0.9500 & $\mathrm{C} 11-\mathrm{H} 11$ & 0.9500 \\
\hline $\mathrm{C} 4-\mathrm{C} 5$ & $1.385(4)$ & $\mathrm{C} 12-\mathrm{C} 13$ & $1.390(4)$ \\
\hline $\mathrm{C} 4-\mathrm{H} 4$ & 0.9500 & $\mathrm{C} 12-\mathrm{H} 12$ & 0.9500 \\
\hline $\mathrm{C} 5-\mathrm{C} 6$ & $1.372(4)$ & $\mathrm{C} 13-\mathrm{H} 13$ & 0.9500 \\
\hline $\mathrm{C} 2-\mathrm{O} 1-\mathrm{H} 1$ & 105.1 & $\mathrm{~N} 1-\mathrm{C} 7-\mathrm{C} 1$ & $126.9(2)$ \\
\hline $\mathrm{C} 7-\mathrm{N} 1-\mathrm{O} 2$ & $122.78(18)$ & $\mathrm{N} 1-\mathrm{C} 7-\mathrm{H} 7$ & 116.6 \\
\hline $\mathrm{C} 7-\mathrm{N} 1-\mathrm{C} 8$ & $121.79(18)$ & $\mathrm{C} 1-\mathrm{C} 7-\mathrm{H} 7$ & 116.6 \\
\hline $\mathrm{O} 2-\mathrm{N} 1-\mathrm{C} 8$ & $115.43(17)$ & $\mathrm{C} 13-\mathrm{C} 8-\mathrm{C} 9$ & $121.2(2)$ \\
\hline $\mathrm{C} 2-\mathrm{C} 1-\mathrm{C} 6$ & $118.6(2)$ & $\mathrm{C} 13-\mathrm{C} 8-\mathrm{N} 1$ & $117.2(2)$ \\
\hline $\mathrm{C} 2-\mathrm{C} 1-\mathrm{C} 7$ & $125.97(19)$ & $\mathrm{C} 9-\mathrm{C} 8-\mathrm{N} 1$ & $121.66(19)$ \\
\hline $\mathrm{C} 6-\mathrm{C} 1-\mathrm{C} 7$ & $115.4(2)$ & $\mathrm{C} 10-\mathrm{C} 9-\mathrm{C} 8$ & $118.9(2)$ \\
\hline $\mathrm{O} 1-\mathrm{C} 2-\mathrm{C} 3$ & $118.3(2)$ & $\mathrm{C} 10-\mathrm{C} 9-\mathrm{H} 9$ & 120.5 \\
\hline $\mathrm{O} 1-\mathrm{C} 2-\mathrm{C} 1$ & $122.5(2)$ & $\mathrm{C} 8-\mathrm{C} 9-\mathrm{H} 9$ & 120.5 \\
\hline $\mathrm{C} 3-\mathrm{C} 2-\mathrm{C} 1$ & $119.1(2)$ & $\mathrm{C} 9-\mathrm{C} 10-\mathrm{C} 11$ & $120.6(2)$ \\
\hline $\mathrm{C} 4-\mathrm{C} 3-\mathrm{C} 2$ & $120.8(2)$ & $\mathrm{C} 9-\mathrm{C} 10-\mathrm{H} 10$ & 119.7 \\
\hline $\mathrm{C} 4-\mathrm{C} 3-\mathrm{H} 3$ & 119.6 & $\mathrm{C} 11-\mathrm{C} 10-\mathrm{H} 10$ & 119.7 \\
\hline $\mathrm{C} 2-\mathrm{C} 3-\mathrm{H} 3$ & 119.6 & $\mathrm{C} 12-\mathrm{C} 11-\mathrm{C} 10$ & $119.6(2)$ \\
\hline $\mathrm{C} 3-\mathrm{C} 4-\mathrm{C} 5$ & $120.5(2)$ & $\mathrm{C} 12-\mathrm{C} 11-\mathrm{H} 11$ & 120.2 \\
\hline $\mathrm{C} 3-\mathrm{C} 4-\mathrm{H} 4$ & 119.7 & $\mathrm{C} 10-\mathrm{C} 11-\mathrm{H} 11$ & 120.2 \\
\hline $\mathrm{C} 5-\mathrm{C} 4-\mathrm{H} 4$ & 119.7 & $\mathrm{C} 11-\mathrm{C} 12-\mathrm{C} 13$ & $120.5(2)$ \\
\hline $\mathrm{C} 6-\mathrm{C} 5-\mathrm{C} 4$ & $119.6(2)$ & $\mathrm{C} 11-\mathrm{C} 12-\mathrm{H} 12$ & 119.8 \\
\hline $\mathrm{C} 6-\mathrm{C} 5-\mathrm{H} 5$ & 120.2 & $\mathrm{C} 13-\mathrm{C} 12-\mathrm{H} 12$ & 119.8 \\
\hline
\end{tabular}




$\begin{array}{llll}\mathrm{C} 4-\mathrm{C} 5-\mathrm{H} 5 & 120.2 & \mathrm{C} 8-\mathrm{C} 13-\mathrm{C} 12 & 119.2(2) \\ \mathrm{C} 5-\mathrm{C} 6-\mathrm{C} 1 & 121.2(2) & \mathrm{C} 8-\mathrm{C} 13-\mathrm{H} 13 & 120.4 \\ \mathrm{C} 5-\mathrm{C} 6-\mathrm{H} 6 & 119.4 & \mathrm{C} 12-\mathrm{C} 13-\mathrm{H} 13 & 120.4 \\ \mathrm{C} 1-\mathrm{C} 6-\mathrm{H} 6 & 119.4 & & \\ & & & \\ \mathrm{C} 6-\mathrm{C} 1-\mathrm{C} 2-\mathrm{O} 1 & 172.1(2) & \mathrm{C} 6-\mathrm{C} 1-\mathrm{C} 7-\mathrm{N} 1 & 153.4(2) \\ \mathrm{C} 7-\mathrm{C} 1-\mathrm{C} 2-\mathrm{O} 1 & -4.2(3) & \mathrm{C} 7-\mathrm{N} 1-\mathrm{C} 8-\mathrm{C} 13 & -153.0(2) \\ \mathrm{C} 6-\mathrm{C} 1-\mathrm{C} 2-\mathrm{C} 3 & -4.4(3) & \mathrm{O} 2-\mathrm{N} 1-\mathrm{C} 8-\mathrm{C} 13 & 27.7(3) \\ \mathrm{C} 7-\mathrm{C} 1-\mathrm{C} 2-\mathrm{C} 3 & 179.3(2) & \mathrm{C} 7-\mathrm{N} 1-\mathrm{C} 8-\mathrm{C} 9 & 27.3(3) \\ \mathrm{O} 1-\mathrm{C} 2-\mathrm{C} 3-\mathrm{C} 4 & -174.0(2) & \mathrm{O} 2-\mathrm{N} 1-\mathrm{C} 8-\mathrm{C} 9 & -152.0(2) \\ \mathrm{C} 1-\mathrm{C} 2-\mathrm{C} 3-\mathrm{C} 4 & 2.6(3) & \mathrm{C} 13-\mathrm{C} 8-\mathrm{C} 9-\mathrm{C} 10 & -0.1(3) \\ \mathrm{C} 2-\mathrm{C} 3-\mathrm{C} 4-\mathrm{C} 5 & 0.1(4) & \mathrm{N} 1-\mathrm{C} 8-\mathrm{C} 9-\mathrm{C} 10 & 179.6(2) \\ \mathrm{C} 3-\mathrm{C} 4-\mathrm{C} 5-\mathrm{C} 6 & -1.0(4) & \mathrm{C} 9-\mathrm{C} 9-\mathrm{C} 10-\mathrm{C} 11 & -0.2(4) \\ \mathrm{C} 4-\mathrm{C} 5-\mathrm{C} 6-\mathrm{C} 1 & -0.9(4) & \mathrm{C} 10-\mathrm{C} 11-\mathrm{C} 12-\mathrm{C} 12 & 0.6(4) \\ \mathrm{C} 2-\mathrm{C} 1-\mathrm{C} 6-\mathrm{C} 5 & 3.6(3) & \mathrm{C} 9-\mathrm{C} 8-\mathrm{C} 13-\mathrm{C} 12 & -0.7(4) \\ \mathrm{C} 7-\mathrm{C} 1-\mathrm{C} 6-\mathrm{C} 5 & -179.7(2) & \mathrm{N} 1-\mathrm{C} 8-\mathrm{C} 13-\mathrm{C} 12 & 0.0(3) \\ \mathrm{O} 2-\mathrm{N} 1-\mathrm{C} 7-\mathrm{C} 1 & -0.4(3) & \mathrm{C} 11-\mathrm{C} 12-\mathrm{C} 13-\mathrm{C} 8 & -179.8(2) \\ \mathrm{C} 8-\mathrm{N} 1-\mathrm{C} 7-\mathrm{C} 1 & -179.7(2) & & 0.4(4) \\ \mathrm{C} 2-\mathrm{C} 1-\mathrm{C} 7-\mathrm{N} 1 & -30.2(3) & & \end{array}$

Hydrogen-bond geometry $\left(A,{ }^{\circ}\right)$

$C g 1$ and $C g 2$ are the centroids of the $\mathrm{C} 1-\mathrm{C} 6$ and $\mathrm{C} 8-\mathrm{C} 13$ aromatic rings, respectively.

\begin{tabular}{lllll}
\hline$D-\mathrm{H} \cdots A$ & $D-\mathrm{H}$ & $\mathrm{H} \cdots A$ & $D \cdots A$ & $D-\mathrm{H} \cdots A$ \\
\hline $\mathrm{O} 1-\mathrm{H} 1 \cdots \mathrm{O} 2$ & 0.97 & 1.53 & $2.479(2)$ & 167 \\
$\mathrm{C} 7-\mathrm{H} 7 \cdots \mathrm{O} 2^{\mathrm{i}}$ & 0.95 & 2.43 & $3.368(3)$ & 167 \\
$\mathrm{C} 10-\mathrm{H} 10 \cdots \mathrm{O} 1^{\mathrm{ii}}$ & 0.95 & 2.53 & $3.227(3)$ & 131 \\
$\mathrm{C} 11-\mathrm{H} 11 \cdots C g 1^{\mathrm{iii}}$ & 0.95 & 2.94 & $3.662(3)$ & 136 \\
$\mathrm{C} 4-\mathrm{H} 4 \cdots \mathrm{Cg} 2^{\mathrm{iv}}$ & 0.95 & 2.77 & $3.545(3)$ & 140
\end{tabular}

Symmetry codes: (i) $x-1, y, z$; (ii) $x-1,-y, z-1 / 2$; (iii) $x,-y, z-1 / 2$; (iv) $x,-y+1, z+1 / 2$. 